J. Indones. Math. Soc. (MIHMI)

Vol. 15, No. 2 (2009), pp. 97-104.

\title{
PARAMETRIC EXCITATION IN A SELF-EXCITED THREE-DEGREES OF FREEDOM PROBLEM
}

\author{
SITI FATIMAH
}

\begin{abstract}
The effect of parametric excitation in self-excited has been investigated in two-degrees of freedom problems. The possibility of suppressing self-excited vibrations by using parametric excitation and the dynamic behavior of those kind systems were discussed. In the this paper, we consider a system in three-degrees of freedom problem which by using a linear transformation the system becomes an Autoparametric. The system consists of a central mass and two external masses where those masses are conected by springs with the same constant stiffness. The flow-generated self-excited force is acting on the external masses, it is represented by Rayleigh force. The variable stiffness is periodically varying in time, represents a parametric excitation. It turns out that for certain parameter ranges full vibration cancellation is possible. The analysis of linear case of system shows that there are two conditions in order to obtain an interval of the parametric excitation. Using the averaging method the fully non-linear system is investigated producing as non-trivial solutions unstable periodic solutions. The behavior of this unstable solution is studied in the full system.
\end{abstract}

\section{INTRODUCTION}

Some slender structures, like tubes in heat exchangers or rods between two walls, are often self-excited by flow. In these cases, the self-excited vibration is an unfavorable phenomenon leading to an increased loading of structural elements. It is sometimes able to break down them. The system can be realized by a mechatronic device similar to magnetic bearings or by pneumatic springs with a variable inner pressure, see Tondl and Nabergoj [9]. There are several ways to limit the influence

Received 30-10-2009, Accepted 20-12-2009.

2000 Mathematics Subject Classification: 37C99

Key words and Phrases: Parametric Excitation, autoparametric, Three-degrees of freedom problems 
of self-excited vibrations; for instance, using a conventional spring-mass absorber or different kinds of damping; see Tondl [10], [12], [13] for references.

The effect of parametric excitation has been investigated in a two-mass system, see Tondl [9], [14], [15] and Fatimah and Verhulst [2], [3]. In Ecker and Tondl [1], the conditions when full suppression of the vibration could be achieved, have been analytically studied. In Fatimah and Verhulst [3], the same system as in [1] is considered. The results show that, using the first-order approximation of the averaging method, full vibration cancellation is possible in an open parameter set. Using the second-order approximation, the minimum value of the response can be calculated if the secondary mass is small. It shows that a large amount of quenching is still possible. The results of the influence of the parametric excitation on the self-excitation in two-degrees of freedom system suggest to study its contribution in higher degree of freedom systems.

In this study we consider a three-degrees of freedom system governed by the following differential equations:

$$
\begin{gathered}
m_{1} \ddot{y}_{1}+k\left(2 y_{1}-y_{2}\right)-b U^{2}\left(1-\gamma_{o} \dot{y}_{1}^{2}\right) \dot{y}_{1}=0 \\
m \ddot{y}_{2}+k\left(2 y_{2}-\left(y_{1}+y_{3}\right)\right)+k_{o}(1+\varepsilon \cos \omega t) y_{2}+b_{o} \dot{y}_{2}=0 \\
m_{2} \ddot{y}_{3}+k\left(2 y_{3}-y_{2}\right)-b U^{2}\left(1-\gamma_{\circ} \dot{y}_{3}^{2}\right) \dot{y}_{3}=0 .
\end{gathered}
$$

The simple model of this system is shown in the Figure 1. The model consists of a central mass and two external masses. The ends of the external masses are elastically mounted by using springs of variable stiffness. The central mass $m$ and the external masses $m_{1}$ and $m_{2}$ represent reduced concentrated masses of body elements while the connecting springs simulate their elasticity. The springs have constant stiffness $k$. The flow-generated self-excited force is acting on the external masses $m_{1}$ and $m_{2}$; it is represented by Rayleigh force in the form $b U(1-$ $\left.\gamma_{o} \dot{y}_{i}^{2}\right) \dot{y}_{i}, i=1,3$, where $b$ and $\gamma_{o}$ are positive coefficients and $U$ is the flow velocity. The linear viscous damping of the central mass $m$ has coefficient $b_{o}$, while the variable stiffness is periodically varying in time as $k_{o}(1+\varepsilon \cos \omega t)$.

The system (3) can be transformed into a standard form which is an $\mathrm{Au}-$ toparametric system. There exist a large number of studies of similar autoparametric systems. In Ruijgrok [6], Queini, Chin, and Nayfeh [5], Tien, Namachchivaya, and Bajaj [8], and Fatimah and Ruijgrok [4], the bifurcations of the averaged system are studied in combination with external excitation. The results show analytically that for certain values of the parameters, a Silnikov bifurcation can occur, leading to chaotic solutions.

The purpose of this paper is to study the behavior of the semi-trivial solution of system (3). This is done by using the method of averaging. 


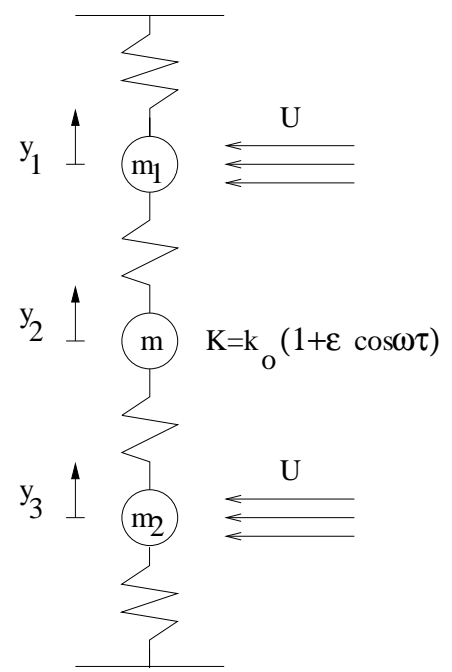

Figure 1: The schematic representation of the three-mass chain system.

\section{ANALYSIS OF MODEL}

The system (3) is studied for the case $m_{1}=m_{2}=m$. Using the time transformation $\tau=\omega_{o} t$ with $\omega_{o}=\sqrt{2 k / m}$, system (3) becomes

$$
\begin{aligned}
y_{1}^{\prime \prime}+y_{1}-\frac{1}{2} y_{2}-\varepsilon \beta V^{2}\left(1-\gamma y_{1}^{\prime 2}\right) y_{1}^{\prime} & =0 \\
y_{2}^{\prime \prime}+y_{2}-\frac{1}{2}\left(y_{1}+y_{3}\right)+q^{2}(1+\varepsilon \cos \eta \tau) y_{2}+\varepsilon \mu y_{2}^{\prime} & =0 \\
y_{3}^{\prime \prime}+y_{3}-\frac{1}{2} y_{2}-\varepsilon \beta V^{2}\left(1-\gamma y_{3}^{\prime 2}\right) y_{3}^{\prime} & =0
\end{aligned}
$$

where

$$
\varepsilon \beta=\frac{b / m}{\omega_{o}}, \quad \eta=\frac{\omega}{\omega_{o}}, \quad q^{2}=\frac{k_{o} / m}{\omega_{o}^{2}} U^{2}, \quad \varepsilon \mu=\frac{b_{o} / m}{\omega_{o}}, \quad \gamma=\gamma_{o} \omega_{o}^{2}, \text { and } V=\frac{U}{U_{o}}
$$

Using the linear transformation

$$
\begin{aligned}
& y_{1}=x_{1}+x_{2}+x_{3} \\
& y_{2}=a_{1} x_{1}+a_{2} x_{2} \\
& y_{3}=x_{1}+x_{2}-x_{3},
\end{aligned}
$$

system (4) can be transformed into a standard form (7).

$$
\begin{array}{r}
x_{1}^{\prime \prime}+\Omega_{1}^{2} x_{1}+\varepsilon f_{1}\left(\alpha_{1}, \cos \eta \tau, \mathbf{x}\right)=0 \\
x_{2}^{\prime \prime}+\Omega_{2}^{2} x_{2}+\varepsilon f_{2}\left(\alpha_{2}, \cos \eta \tau, \mathbf{x}\right)=0 \\
x_{3}^{\prime \prime}+x_{3}+\varepsilon f_{3}\left(\theta_{31}, \mathbf{x}\right)=0
\end{array}
$$


where

$$
\mathbf{x}=\left(x_{i}, i=1,2,3\right), \quad \alpha_{i}=\left(\theta_{i j}, Q_{i j} ; i=1,2, j=1,2\right) .
$$

The $x_{i}, i=1,2,3$ are the normal coordinates corresponding to free vibrations of the system. The normal-mode frequencies $\Omega_{1,2}$ and the constant multipliers $a_{1,2}$ are given by the relations:

$$
\begin{aligned}
\Omega_{1,2} & =\frac{1}{2}\left(q^{2}+2\right) \mp \frac{1}{2} \sqrt{q^{4}+2}, \\
a_{1,2} & =-q^{2} \pm \sqrt{q^{4}+2},
\end{aligned}
$$

where $q \neq 0$ and $q \neq 1$. We note that $\Omega_{2}>\Omega_{1}>0, a_{1}>0, a_{2}<0$ and

$$
\begin{aligned}
f_{i} & =\frac{1}{2\left(a_{1}-a_{2}\right)}\left(\theta_{i 1} x_{1}^{\prime}+\theta_{i 2} x_{2}^{\prime}+\left(Q_{i 1} x_{1}+Q_{i 2} x_{2}\right) \cos \eta \tau\right. \\
& \left.\mp 2 a_{2,1} \beta V^{2} \gamma\left(3\left(x_{1}^{\prime}+x_{2}^{\prime}\right)^{2} x_{3}^{\prime}+\left(x_{1}^{\prime}+x_{2}^{\prime}\right)^{3}\right)\right), i=1,2 . \\
f_{3} & =\frac{1}{2\left(a_{1}-a_{2}\right)}\left(\theta_{31} x_{3}^{\prime}+4\left(a_{1}-a_{2}\right) \beta V^{2} \gamma\left(x_{3}^{\prime}{ }^{3}+x_{3}^{\prime}\left(x_{1}^{\prime}+x_{2}^{\prime}\right)^{2}\right)\right.
\end{aligned}
$$

We note that system (9) is an Autoparametric system where $x_{3}=0$ corresponds to the semi-trivial solution of the system. Next, we use the parameters $\theta_{11}, \theta_{22}, Q_{12}$, and $Q_{21}$, where

$$
\begin{array}{ll}
\theta_{11}=2\left(a_{1} \mu+a_{2} \beta V^{2}\right), & \theta_{22}=-2\left(a_{2} \mu+a_{1} \beta V^{2}\right) \\
\theta_{31}=-2\left(a_{1}-a_{2}\right) \beta V^{2}, & Q_{12}=2 q^{2} a_{2}, \quad Q_{21}=-2 q^{2} a_{1}
\end{array}
$$

For the initiation of self-excited vibration either $\theta_{11}$ or $\theta_{22}$ (or both) must be negative. Thus, there exist two conditions in order to obtain an interval of $\eta$ where the trivial solution of system (7) is stable. The first condition is

$$
\theta_{11}+\theta_{22}>0
$$

and the second condition for the stability interval boundaries is

$$
\eta_{\circ}-\Delta<\eta<\eta_{\circ}+\Delta
$$

where $\Delta=\varepsilon g\left(\theta_{i i}, Q_{i j}, \Omega_{i}\right), i, j=1,2$.

\section{THE NORMAL FORM BY AVERAGING}

We note that the parametric excitation terms only occur in the normal coordinates $x_{1}, x_{2}$. There are three natural frequencies of system (7), i.e, $\Omega_{1}, \Omega_{2}$, and $\Omega_{3}=1$. Due to occurrence of parametric resonance and self-excitation of system (7), we consider the external resonance $\eta=\Omega_{2}-\Omega_{1}$ and the internal resonance $\Omega_{2}-\Omega_{3}-2 \Omega_{1}=0$. Transforming $t \rightarrow \eta \tau$ and allowing detuning near $\eta_{\circ}$ by putting

$$
\eta=\eta_{o}+\varepsilon \bar{\sigma}, \quad \eta_{o}=\Omega_{2}-\Omega_{1}
$$


we then transform system (7) by using Lagrange transformation,

$$
\begin{aligned}
& x_{i}=u_{i} \cos \omega_{i} t+v_{i} \sin \omega_{i} t, \\
& \dot{x}_{1}=-\omega_{i} u_{i} \sin \omega_{i} t+\omega_{i} v_{i} \cos \omega_{i} t,
\end{aligned}
$$

for $i=1,2,3$ and $\omega_{i}=\frac{\Omega_{i}}{\eta}$. We use again the dot to indicate derivation with respect to the re-scaled time. After averaging over $2 \pi$ and then rescaling time through $\frac{\varepsilon}{2\left(a_{1}-a_{2}\right) \eta_{0}^{2}}$, the first order in $\varepsilon$ of the averaged system is of the form;

$$
\dot{\mathbf{U}}=A \mathbf{U}+\mathbf{F}(\mathbf{U})
$$

where $\mathbf{U}$ is a vector $\left(u_{i}, v_{i}, i=1,2,3\right)$ and $\mathbf{F}$ is a vector function $\left(f_{i}, i=1 . .6\right)$. The function $\mathbf{F}$ only contains a cubic nonlinearity. The constant $6 \times 6$-matrix $A$ is in the form

$$
A=\left(\begin{array}{ccc}
A_{11} & A_{12} & 0 \\
A_{21} & A_{22} & 0 \\
0 & 0 & A_{33}
\end{array}\right)
$$

where $A_{i j}, i, j=1,2, \emptyset$ and $A_{33}$ are $2 \times 2$-matrix. System (15) can be reduced to the five-dimension system by transforming the system using the following transformation,

$$
u_{i}=-R_{i} \cos \psi_{i}, \quad \text { and } \quad v_{i}=R_{i} \sin \psi_{i}, \quad i=1,2,3
$$

This transformation is useful for studying the semitrivial solution $\left(x_{1}, x_{2}, 0\right)$ of system (8) when $x_{1} \neq 0, x_{2} \neq 0$.

\section{THE SEMITRIVIAL SOLUTION}

Consider $\lambda_{i}, i=1 . .6$ which are the eigenvalues of matrix $A$. We find that the real parts of $\lambda_{5}$ and $\lambda_{6}$ of the trivial solution are positive. Then, the trivial solution of system (15) is always unstable. Let $\left(x_{10}, x_{20}, 0\right)$ be a semitrivial solution of system (15), where $x_{10}, x_{20}$ correspond with the non-trivial solutions $R_{10}, R_{20}$, and $\Psi_{0}$ of the following system

$$
\begin{aligned}
& \dot{R_{1}}=-\theta_{11} \eta_{o} R_{1}+\frac{1}{2} \frac{Q_{12}}{\omega_{1}} R_{2} \sin (\Psi)+\frac{3}{2} a_{2} \alpha \eta_{o}^{3} R_{1}\left(\frac{1}{2} \omega_{1}^{2} R_{1}^{2}+\omega_{2}^{2} R_{2}^{2}\right) \\
& \dot{R_{2}}=-\theta_{22} \eta_{\circ} R_{2}-\frac{1}{2} \frac{Q_{21}}{\omega_{2}} R_{1} \sin \Psi-\frac{3}{2} a_{1} \alpha \eta_{o}^{3} R_{2}\left(\omega_{1}^{2} R_{1}^{2}+\frac{1}{2} \omega_{2}^{2} R_{2}^{2}\right) \\
& \dot{\Psi}=2\left(a_{1}-a_{2}\right) \eta_{\circ} \bar{\sigma}+\frac{1}{2}\left(\frac{Q_{21}}{\omega_{2}} \frac{R_{1}}{R_{2}}-\frac{Q_{12}}{\omega_{1}} \frac{R_{2}}{R_{1}}\right) \cos \Psi .
\end{aligned}
$$

When we take $\dot{R}_{1}=0, \dot{R}_{2}=0$, and $\dot{\Psi}=0$, we obtain fixed points of system (18). They correspond with periodic solutions of system (15). 
The fixed points of system (18) for the case $\Psi=0$ are obtained by intersecting the following $z_{1}$ and $z_{2}$.

$$
\begin{aligned}
& z_{1}:\left(a_{1} \theta_{11}-\frac{1}{2} a_{2} \theta_{22}\right) \omega_{1}^{2} R_{1}^{2}-\left(\frac{1}{2} a_{1} \theta_{11}-a_{2} \theta_{22}\right) \omega_{2}^{2} R^{2}=0 \\
& z_{2}: 2\left(a_{1}-a_{2}\right) \eta_{\circ} \sigma \omega_{1} \omega_{2} R_{1} R_{2}+\frac{1}{2}\left(\omega_{1} Q_{21} R_{1}^{2}-\omega_{2} Q_{2} R_{2}^{2}\right)=0 .
\end{aligned}
$$

When $\bar{\sigma}_{2}<\bar{\sigma}<\bar{\sigma}_{1}$ and $\bar{\sigma}_{2}<0$ and $\bar{\sigma}_{1}>0$, then $z_{1} \cap z_{2}=\emptyset$. The explicit expression for $\bar{\sigma}_{i}(i=1,2)$ can be found from equations (3.12) and (3.15).

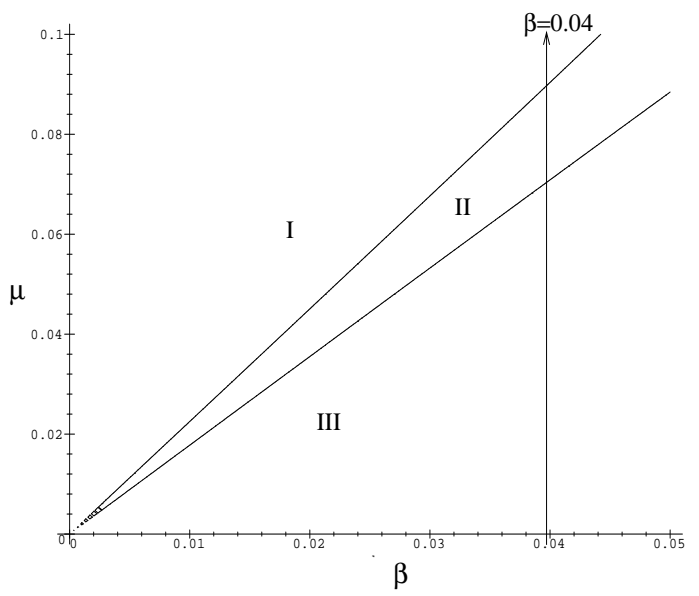

Figure 2: The parameter diagram in the $(\beta, \mu)$-plane for fixed $q=0.85, V=\sqrt{2}$, $\gamma=1$, and $\varepsilon=0.1$ for nontrivial fixed points of system (18). We find that there is no fixed point in region $\mathrm{I}$. One fixed point $\mathbf{R}_{\mathbf{0 1}}$ exists in region II. There are two fixed points $\mathbf{R}_{\mathbf{0 1}}$ and $\mathbf{R}_{\mathbf{0 2}}$ in region III. The line $\beta=0.04$ is used in the numerical example in Figure 3.

Figure 2 shows the existence of the fixed point $\mathbf{R}_{\mathbf{0}}$ when the parameter $\mu$ is varied along line $\beta=0.04$. There is no fixed point of system (18) in region $\mathrm{I}$ for $\mu>0.10736$. The fixed point $\mathbf{R}_{\mathbf{0 1}}^{+}$exists in the interval $0.05987<\mu<0.10736$ in region II. There are two fixed points $\mathbf{R}_{\mathbf{0 1}}^{+}$and $\mathbf{R}_{\mathbf{0 2}}^{-}$in the interval $0.02219<\mu<$ 0.05987 in region III. Two fixed points $\mathbf{R}_{\mathbf{0 1}}^{-}$and $\mathbf{R}_{\mathbf{0 2}}{ }^{-}$exist for $0<\mu<0.02219$. Figure 3 shows the fixed points $\mathbf{R}_{\mathbf{0}}^{+}$and $\mathbf{R}_{\mathbf{0}}^{-}$are attracting and it is non attracting, respectively, in the $\left(R_{1}, R_{2}\right)$-plane. The solid curve shows that the fixed point $\mathbf{R}_{\mathbf{0}}$ is attracting in the $\left(R_{1}, R_{2}\right)$-plane. The dashed curve shows that it is non attracting. We note that the fixed point $\left(\mathbf{R}_{\mathbf{0}}, \mathbf{0}\right)$ is always unstable in the full system. 


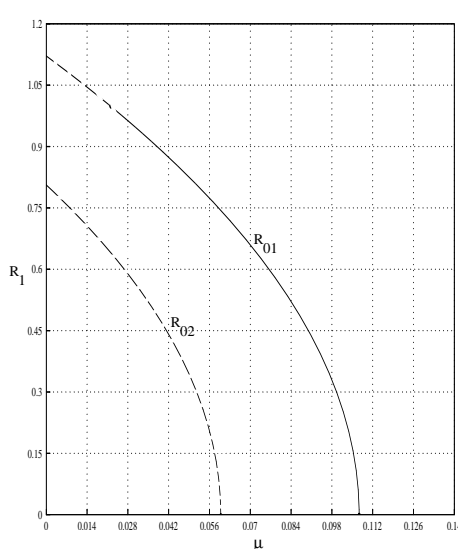

(a)

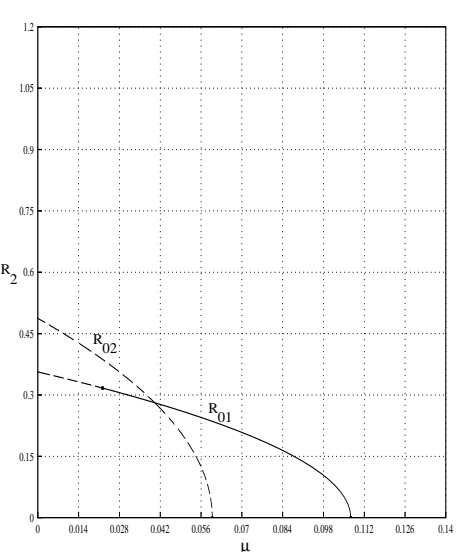

(b)

Figure 3: The existence of the fixed point $\mathbf{R}_{0}$ of system (15), for fixed $q=0.85$, $V=\sqrt{2}, \gamma=1, \varepsilon=0.1$, and $\beta=0.04$, (a) in the $\left(\mu, R_{1}\right)$-plane and (b) in the $\left(\mu, R_{2}\right)$-plane. There is no fixed point for $\mu>0.10736$. There is one solution $\mathbf{R}_{\mathbf{0 1}}^{+}$ for $0.05987<\mu<0.10736$. There are two solutions $\mathbf{R}_{\mathbf{0 1}}^{+}$and $\mathbf{R}_{\mathbf{0 2}}^{-}$in the interval $0.02219<\mu<0.05987$. Two solutions $\mathbf{R}_{\mathbf{0 1}}^{-}$and $\mathbf{R}_{\mathbf{0 2}}{ }^{-}$exist for $0<\mu<0.02219$. The solid curve shows that the solution $\mathbf{R}_{0}$ is attracting in the $\left(R_{1}, R_{2}\right)$-plane. The dashed curve shows that it is non attracting.

In a further study one has to analyze the behavior of this unstable semitrivial solution $\left(\mathbf{R}_{\mathbf{0}}, \mathbf{0}\right)$ in the full system.

\section{CONCLUDING REMARKS}

The analysis of the linear case of the system show that there are two condition in order to obtain an interval of excitation. First condition states that the sum of the negative and the positive linear damping components determine the stability of certain modes and must be positive. The second condition is related to the parametric excitation frequency and determines, whether full quenching can be achieved or not in certain interval. The Figure 2 shows that the system has no semi-trivial solution in region I. A semi-trivial solution exists in region II. There are two semi-trivial solutions in region III.

\section{REFERENCES}

1. H. ECKER, AND A. TONDL, Suppressing of flow-induced Vibrations by a dynamic absorber with Parametric Excitation, Proc. of $7^{\text {th }}$ International Conference on FlowInduced Vibrations FIV2000, Lucerne, Switzerland, 2000. 
2. S. Fatimah And F. Verhulst, Bifurcation in Flow-induced Vibration, Proceeding of the international Conference SPT2002, Symmetry and Perturbation Theory, 19-26 May, ISBN 981-238-241-0, Cala Ganone, Sardinia, Italy, 2002.

3. S. Fatimah and F. Verhulst, "Suppressing flow-induced Vibration by Parametric Excitation", Nonlinear Dynamics Journal 31 (2003), 275-298.

4. S. FAtimah And M. RuiJgrok, "Bifurcations in an autoparametric system in 1:1 internal resonance with parametric excitation", International J. Non-linear Mechanics $\mathbf{3 7 : 2}$ (2002), 297-308.

5. S.S. Oueini, C. Chin, And A.H. Nayfeh, "Response of Two Quadratically Coupled Oscillators to a Principal Parametric Excitation", to Appear J. of Vibration and Control.

6. M. Ruijgrok, Studies in Parametric and Autoparametric Resonance, Ph.D. Thesis, Universiteit Utrecht, 1995.

7. R. Svoboda, A. Tondl, and F. Verhulst, "Autoparametric Resonance by Coupling of Linear and Nonlinear Systems", J. Non-linear Mechanics 29 (1994), 225-232.

8. W. Tien, N.S Namachchivaya, And A.K. Bajaj, "Non-Linear Dynamics of a Shallow Arch under Periodic Excitation-I. 1:2 Internal Resonance", Int. J. Non-Linear Mechanics 29 (1994), 349-366.

9. A. Tondl and R. Nabergoj, "The Effect of Parametric Excitation on a Self-excited Three-mass System", pre-print, 2002.

10. A. Tond, Quenching of Self-excited Vibrations, Elsevier, Prague, 1991.

11. A. Tond, V. Kotek, And C. Kratochvil, Vibration Quenching of Pendulum Type Systems by Means of Absorbers, CERM akademicke nakladatelstvi, s.r.o., Brno, Czech Republic, 2001.

12. A. Tond, "To the interaction of different types of excitations", Proc.of Sem. Interactions and Feedback 9\%, Prague, 25-26 (1997), 111-118.

13. A. Tond, On the Interaction between Self-excited and Parametric Vibrations, Monographs and Memoranda N0.25, National Research Institute for Machine Design, Prague, 1978.

14. A. TOndL, "To the problem of quenching self-excited vibrations", Acta Technica CSAV 43 (1998),109-116.

15. A. TondL, "Two Parametrically Excited Chain Systems", Acta Technica CSAV 47 (2002), 67-74.

16. A. Tond, "Three-Mass self-Excited Systems with Parametric Excitation", Acta Technica CSAV 47 (2002), 165-176.

17. A. Tondl, M. Ruijgrok, F. Verhulst, and R. Nabergoj, Autoparametric Resonance in Mechanical Systems, Cambridge University Press, New York, 2000.

18. V.A. Yakubovich and V.M. Starzhinski, Linear Differential equations With Periodic Coefficienst 2nd ed., John Wiley and Sons, New York, 1975.

Siti Fatimah: Department of Mathematics Education, Indonesia University of Education, Bandung, Indonesia.

E-mail: sitifatimah@upi.edu. 